\title{
Editorial
}

\section{High dilutions and homeopathy}

\section{Saurav Arora}

"Although trained with strict academic standards, researchers have already realized that there is a natural, poorly described and still unexplained phenomenon that involves high dilutions."1 The biomedical community remains critically sceptic to the therapeutic action of homeopathic medicines, which are highly diluted "high dilutions" (HDs). Such HDs are the preferred target of critics, as above Avogadro's limit it is assumed that no molecules remain in a solution.

While HDs defy the dose-response relationship, the clinical experience of homeopaths indicates that the more diluted and agitated the drugs, the more thorough and refined their effects. The effects of HDs have been repeatedly verified in various laboratory models, as well as in clinical practice. In addition to homeopathy, serially diluted and agitated drugs are also used in applied in isopathy, iso-endopathy etc. ${ }^{1}$ More recently, their applicability has extended to new fields, like agronomy.

This new and far-reaching realm of applications of HDs might be represented with a diagram, like the one below:

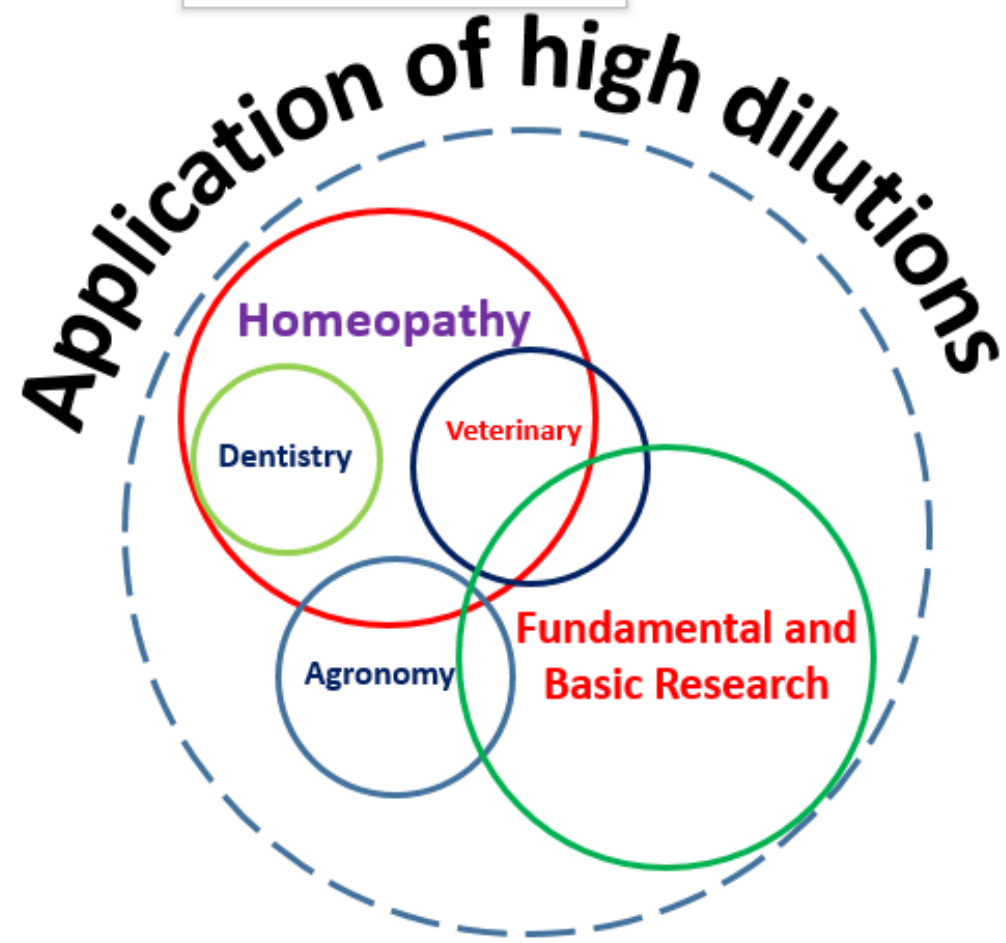

(C) IJHDR I www.highdilution.org 
International Journal of High Dilution

Research (IJHDR) was established to provide an open-access platform to divulgate studies on HDs and its applications. Consistently, for the past six years it published articles on fundamental and basic research, clinical research, veterinary medicine, agronomy, homeopathic pathogenetic trials, literary research including sociological, epistemological and historical research, surveys, debates, education etc. We published about 140 original research articles and five conference proceedings, distributed as depicted in the figure below:

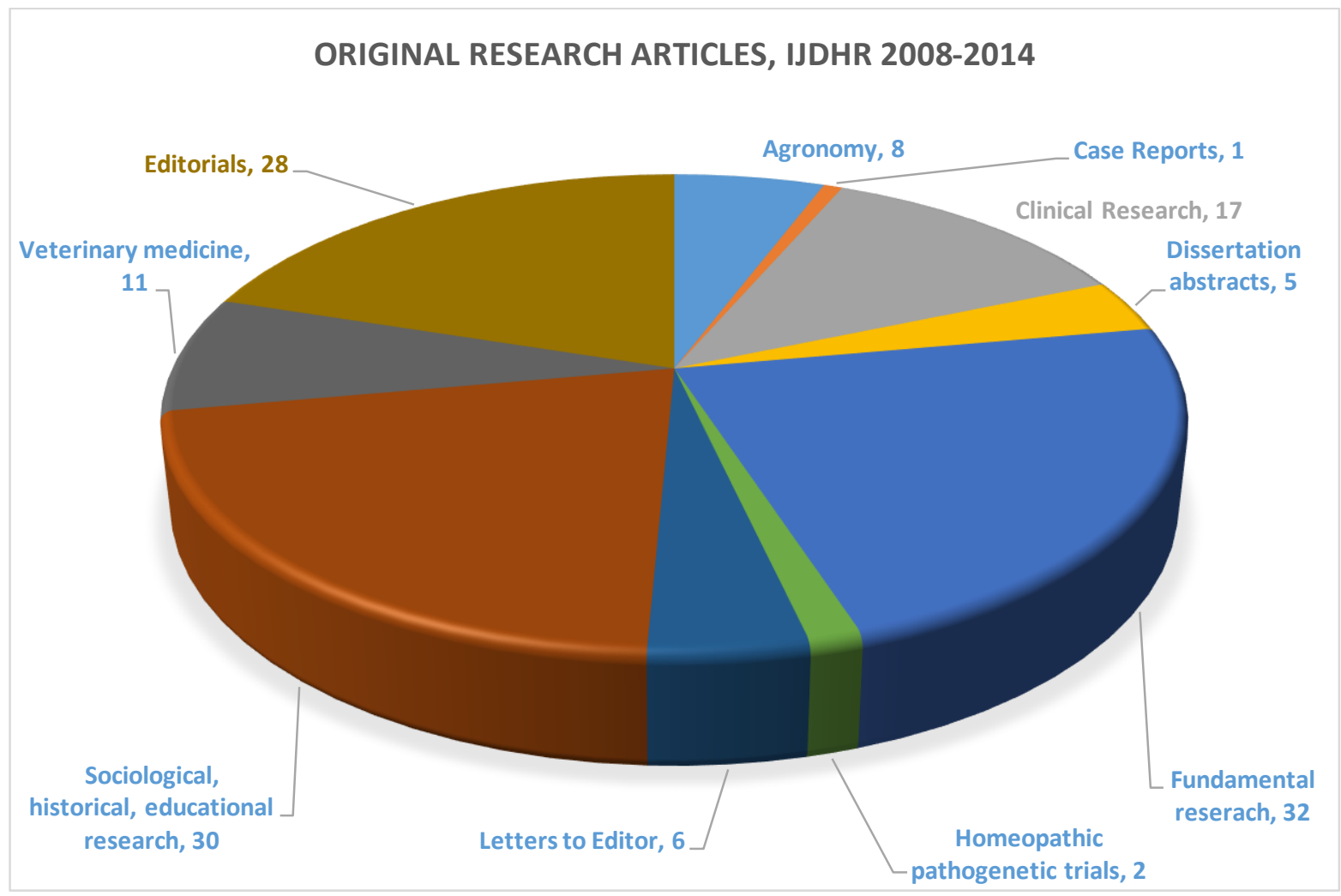

The current issue of IJHDR features a paper by Atheni Konar et al. on the transfer of the effects of HDs of mercuric chloride on $\alpha$ amylase from one test tube to another through capillary water. This study represents the latest in a series previously published by IJHDR and corroborates that water might behave as a carrier of therapeutic information in a cell-free medium.
Further, this issue features two short communications. One, by N. Basu, discusses the usefulness of the homeopathic repertory in the management of chronic suppurative otitis media (CSOM). Basu proposes that the repertory-based approach is superior to prescriptions made without such support. Then, P. Mehra shortly describes the results of an exploratory interventional trial of 
homeopathy in essential hypertension, Reference

which showed that individualized

homeopathy plays a relevant role to offer in

1. Zacharias CR. Publishing: why and the management of this condition. whom to? [Editorial]. Int I High Dilution Res. 2008;7(22): 1-2.

I invite readers to provide feedback on the articles published and to submit their own studies to IJHDR.

(C) International Journal of High Dilution Research.

Not for commercial purposes.

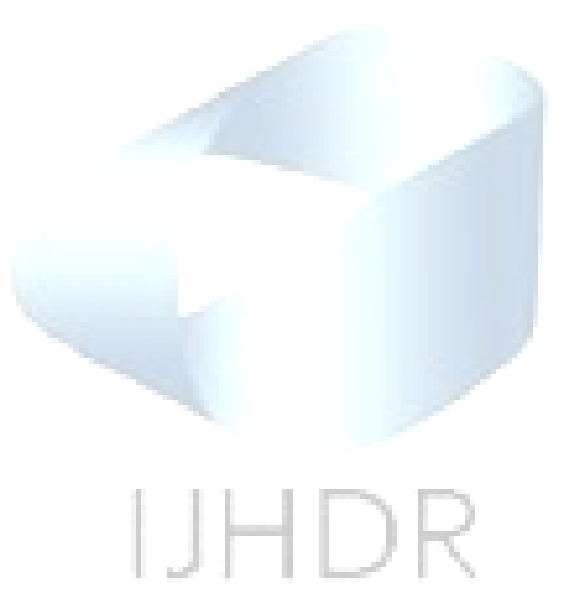

OPEN ACCESS

Cite as: Arora S. High dilutions and homeopathy. Int J High Dilution Res. 2015;14(1):1-3 